\title{
Jefferson.
}

JHN Journal

Volume 13 | Issue 1

Article 1

Winter 2018

\section{Salvage Fractionated Stereotactic Re-irradiation (FSRT) for Patients with Recurrent High Grade Gliomas Progressed after Bevacizumab Treatment}

Wenyin Shi

Thomas Jefferson University, Wenyin.Shi@jefferson.edu

Erik S Blomain

Thomas Jefferson University, erik.blomain@jefferson.edu

Joshua Siglin

University of Pittsburgh Medical Center

Joshua Palmer

Ohio State University

Tu Dan

University of Texas Southwestern

See next page for additional authors

Follow this and additional works at: https://jdc.jefferson.edu/jhnj

Let us know how access to this document benefits you

\section{Recommended Citation}

Shi, Wenyin; Blomain, Erik S; Siglin, Joshua; Palmer, Joshua; Dan, Tu; Wang, Yang; Werner-Wasik, Maria; Glass, MD, Jon; Kim, Lyndon; Bar-Ad, Voichita; Bhamidipati, Deepak; Evans, James J.; Judy, MD, Kevin; Farrell, MD, Christopher J; and Andrews MD, David W. (2018) "Salvage Fractionated Stereotactic Re-irradiation (FSRT) for Patients with Recurrent High Grade Gliomas Progressed after Bevacizumab Treatment," JHN Journal: Vol. 13 : Iss. 1 , Article 1.

DOI: https://doi.org/10.29046/JHNJ.013.1.001

Available at: https://jdc.jefferson.edu/jhnj/vol13/iss1/1

This Article is brought to you for free and open access by the Jefferson Digital Commons. The Jefferson Digital Commons is a service of Thomas Jefferson University's Center for Teaching and Learning (CTL). The Commons is a showcase for Jefferson books and journals, peer-reviewed scholarly publications, unique historical collections from the University archives, and teaching tools. The Jefferson Digital Commons allows researchers and interested readers anywhere in the world to learn about and keep up to date with Jefferson scholarship. This article has been accepted for inclusion in JHN Journal by an authorized administrator of the Jefferson Digital Commons. For more information, please contact:

JeffersonDigitalCommons@jefferson.edu. 


\title{
Salvage Fractionated Stereotactic Re-irradiation (FSRT) for Patients with Recurrent High Grade Gliomas Progressed after Bevacizumab Treatment
}

\author{
Authors \\ Wenyin Shi; Erik S Blomain; Joshua Siglin; Joshua Palmer; Tu Dan; Yang Wang; Maria Werner-Wasik; Jon \\ Glass, MD; Lyndon Kim; Voichita Bar-Ad; Deepak Bhamidipati; James J. Evans; Kevin Judy, MD; \\ Christopher J Farrell, MD; and David W. Andrews MD
}




\section{Salvage Fractionated Stereotactic Re-irradiation (FSRT) for Patients with Recurrent High Grade Gliomas Progressed after Bevacizumab Treatment}

\author{
Wenyin Shi, MD, PhD; Erik S. Blomain, BA²; Joshua Siglin, $\mathrm{MD}^{3}$; Joshua Palmer, \\ MD'; Tu Dan, MD5; Yang Wang, MD ${ }^{6}$; Maria Werner-Wasik, MD ${ }^{1}$; Jon Glass, MD7; \\ Lyndon Kim, MD7; Voichita Bar Ad, MD; Deepak Bhamidipati, BS ${ }^{8}$; James J. \\ Evans, MD7; Kevin Judy, MD7; Christopher Farrell, MD7; David W. Andrews, MD7 \\ ${ }^{1}$ Department of Radiation Oncology, Thomas Jefferson University, Sidney Kimmel Cancer \\ Center at Jefferson, Philadelphia, PA \\ 2Jefferson College of Population Health, Thomas Jefferson University, Philadelphia, PA \\ ${ }^{3}$ Department of Radiation Oncology, University of Pittsburgh Medical Center, Altoona \\ Cancer Center, Altoona, PA \\ ${ }^{4}$ Department of Radiation Oncology, Ohio State University, Columbus, $\mathrm{OH}$ \\ ${ }^{5}$ Department of Radiation Oncology, University of Texas Southwestern, Dallas, TX \\ ${ }^{6}$ Cyberknife Center, Huashan Hospital Pudong, Fudan University, Shanghai, China \\ ${ }^{7}$ Department of Neurological Surgery, Thomas Jefferson University, Philadelphia, PA \\ ${ }^{8}$ Sidney Kimmel Medical College, Thomas Jefferson University, Philadelphia, PA
}

\section{Running title \\ FSRT for recurrent high grade gliomas after avastin failure}

Conflict of Interest Notification

No actual or potential conflicts of interest.

\section{Acknowledgements}

E.S.B. received an F30 Ruth Kirschstein MD-PhD Fellowship Award (CA180500).

\begin{abstract}
Purpose/Objectives: Bevacizumab failure is a major clinical problem in the management of high grade gliomas (HGG), with a median overall survival of less than 4 months $(\mathrm{m})$. This study evaluated the efficacy of fractionated stereotactic re-irradiation (FSRT) for patients with HGG after progression on Bevacizumab.
\end{abstract}

Materials/Methods: Retrospective review was conducted of patients treated with FSRT after progression on bevacizumab. A total of 36 patients were identified. FSRT was most commonly delivered in 3.5 Gy fractions to a total dose of 35 Gy. Survival from initial diagnosis, as well as from recurrence and re-irradiation, were utilized as study endpoints. Univariate and multivariate analysis was performed.

Results: Among the 36 patients, 31 patients had recurrent glioblastoma, and 5 patients had recurrent anaplastic astrocytoma. The median time from initial bevacizumab treatment to FSRT was $8.5 \mathrm{~m}$ (range 2.3 - $32.0 \mathrm{~m}$ ). The median plan target volume for FSRT was $27.5 \mathrm{cc}$ (range 1.95 - $165 \mathrm{cc}$ ). With a median follow up of $20.4 \mathrm{~m}$, the overall survival of the patients since initial diagnosis was also $24.9 \mathrm{~m}$. The median overall survival after initiation of bevacizumab was 13.4 months. The median overall survival from FSRT was $4.8 \mathrm{~m}$. FSRT treatment was well tolerated with no Grade $>3$ toxicity.

Conclusions: Favorable outcomes were observed in patients with recurrent HGG who received salvage FSRT after bevacizumab failure. The treatment was well tolerated. Prospective study is warranted to further evaluate the efficacy of salvage FSRT for selected patients with recurrent HGG amenable to FSRT, who had failed bevacizumab treatment.

\section{INTRODUCTION}

Malignant gliomas are the most common brain tumors, with an estimated yearly incidence of 3 per 100,000 people in the United States. ${ }^{1}$ Despite multiple modalities for definitive therapy (which include resection, radiation therapy (RT) and chemotherapy), these lesions have an unfortunately high rate of recurrence. ${ }^{2}$ Therefore, even despite recent treatment advances in targeted therapies for glioblastoma (GBM) and high grade glioma (HGG) such as bevacizumab, the long-term outcomes for these patients remain poor

In modern clinical practice, treatment failure of recurrent HGGs largely represents failure of bevacizumab therapy. ${ }^{3}$ In addition to disease recurrence itself driving poor outcomes, there is evidence that these patients who fail bevacizumab also harbor disease that is resistant to other systemic therapies. ${ }^{4}$ Therefore, treatment options for these recurrent patients remain limited and their prognosis is dismal with a recent review of sixteen studies reporting an overall survival (OS) of under 4 months after bevacizumab failure. ${ }^{4-6}$

Multiple modalities of radiotherapy have been investigated for this population, including stereotactic radiosurgery (SRS) and brachytherapy. These interventions have been shown to have modest utility, but with the potential for significant associated toxicity. In that context, fractionated stereotactic radiotherapy (FSRT) is a promising treatment modality for the treatment of these refractory HGGs. This modality possesses the precise targeting advantages of SRS but with the dosesparing radiobiologic properties of fractionation to allow greater sparing of surrounding critical structures, thus limiting toxicity.7.8 Taken together, FSRT therefore possesses the potential for decreased toxicity as compared to SRS while still providing excellent local control. ${ }^{9}$ The present study sought 
to evaluate the safety and efficacy of FSRT in patients who failed therapy with bevacizumab.

\section{MATERIALS AND METHODS}

\section{Patients}

The Thomas Jefferson University institutional review board approved this single-institution, retrospective study. Patients who received FSRT salvage after progression on bevacizumab were included. A total of 36 patients were identified from 2006 to 2013. Patients who received FSRT within 2 months of initiation of bevacizumab were excluded. Patients were followed with MRI scans and clinical assessment, which were obtained 6 to 8 weeks after FSRT and at approximately 2-month intervals thereafter.

\section{Treatment Planning}

Treatment decisions were based on consensus recommendations following discussion in our institution's multidisciplinary brain tumor board consisting of radiologists, neurosurgeons, neuro-oncologists, neuropathologists and radiation oncologists. Prior to 2004, treatment planning was conducted with the X-knife 3-D planning system (Radionics, Burlington, MA, USA), which delivered 6 MV photons with a dedicated stereotactic 600SR linear accelerator (Varian, Palo Alto, CA, USA). From 2004 to 2013, treatment planning was carried out with Brain Lab (Novalis) using $\mathrm{mMLC}$ leaves with a leave thickness of $3 \mathrm{~mm}$ and Exac Trac on board imaging. All patients undergoing irradiation were fitted with custom-made Brainlab (Munich, Germany) thermal plastic masks for immobilization. Treatment planning MRI and computed tomography (CT) images were obtained and fused. All patients had thin cut (1-1.5 mm) fat suppressed coronal post-contrast MRI. The gross tumor volume (GTV) was defined on MRI using the gadolinium enhanced T1 weighted series, as peripherally enhancing tissue. Surrounding edema was not purposely included in the treatment volume. The planning target volume was the GTV with minimum margin (0-2 $\mathrm{mm}$ per the treating physician). Critical normal structures, such as optic nerves, chiasm, and brainstem were also contoured. Treatment planning was carried out with Brain Lab Iplan (Munich, Germany). The radiation planning used dynamic conformal arcs, IMRT

Table 1. Patient Demographic and Treatment Information. Descriptive data on our study cohort is shown, encompassing demographic data, clinical information and treatment information.

\begin{tabular}{|l|l|}
\hline Number of patients & 36 \\
\hline Gender & 17 \\
\hline Male & 19 \\
\hline Female & 56 years (37-73) \\
\hline Median Age at FSRT salvage (range) & 80 (50-100) \\
\hline Median KPS at FSRT salvage (range) & \\
\hline Histology at Recurrence & 30 \\
\hline GBM & $4(3-4)$ \\
\hline Anaplastic glioma & $2(1-2)$ \\
\hline Other & 27.5 cc (1.95-165) \\
\hline Median Volume of Recurrence (range) & 35 Gy (30-37.5) \\
\hline Median Radiation Dose (range) & $(2.4-32.1)$ \\
\hline Median time from Bev to FSRT (range) & \\
\hline
\end{tabular}

(intensity modulated radiation therapy) or hybrid-Arcs (Brainlab, Munich, Germany), a combination of dynamic arcs with IMRT beams. The patients were treated with FSRT to a median PTV dose of 35 Gy delivered in 3.5 Gy fractions. ${ }^{10}$ The dose was reduced to 30 Gy in 3 Gy fractions for large targets, and high critical normal structure dose. The constraints for normal critical structures include: brainstem max dose <20 Gy; optic nerve max dose < 15 Gy, chiasm max dose < 15 Gy.

\section{Statistical analysis}

The primary end point of the study was overall survival from initial diagnosis, as well as survival from first recurrence and re-irradiation (described in greater detail under Statistical Analyses). Toxicity was also graded using Radiation Therapy Oncology Group (RTOG) criteria. Overall survival was defined as the time from initial diagnosis to the time of death. Date of recurrence was defined as the date of radiographic evidence of progression. Survival from recurrence and from reirradiation were therefore defined as the time from this radiologic evidence or radiation therapy until death, respectively. Kaplan-Meier curves were generated for the overall survival endpoint. Cox proportional hazard modeling was used for multivariate analysis with factors analyzed in a step-wise fashion. All statistical analysis was performed using the STATA data analysis and statistical software version 13.1 (STATA Corporation).

\section{RESULTS}

\section{Patient Population and Treatment Parameters}

We identified 36 patients with either anaplastic astrocytoma (5 patients) or glioblastoma multiforme (GBM) (30 patients) who had clinical and radiographic evidence of tumor progression on bevacizumab and received FSRT between 2006 and 2013 (Table 1). One patient had gemistocytic astrocytoma. Patient characteristics are listed in Table 1. There were 17 males and 19 females. All patients received initial surgery and were treated with radiation and temozolomide. The median age at recurrence was 57.1 years (range 37-73). The median Karnofsky Performance Status at recurrence was $80 \%$. Following disease progression on bevacizumab, the median target volume treated with FSRT was $27.5 \mathrm{cc}$ (range 1.95 - $165 \mathrm{cc}$ ). The median dose was 35 Gy (range, 30 Gy - 37.5 Gy).

\section{Survival}

Patients underwent routine surveillance for a median follow up of $20.4 \mathrm{~m}$ after initial diagnosis, with an overall survival from initial diagnosis of $24.9 \mathrm{~m}$. Upon evidence of initial disease recurrence, patients were promptly started on bevacizumab. The median overall survival after initiation of bevacizumab was 13.4 months. The median time from initial bevacizumab treatment to initiation of 
Table 2. Survival Statistics. Survival data accrued from the study cohort is shown, expressed as OS from diagnosis, recurrence and from FSRT.

\begin{tabular}{|l|l|}
\hline Median Overall Survival (Range) & 24.9 months (11.4-94.2) \\
\hline From Diagnosis & 12.0 months (4.2-49.1) \\
\hline From First Recurrence & 4.8 months (0.5-23.4) \\
\hline From FSRT &
\end{tabular}

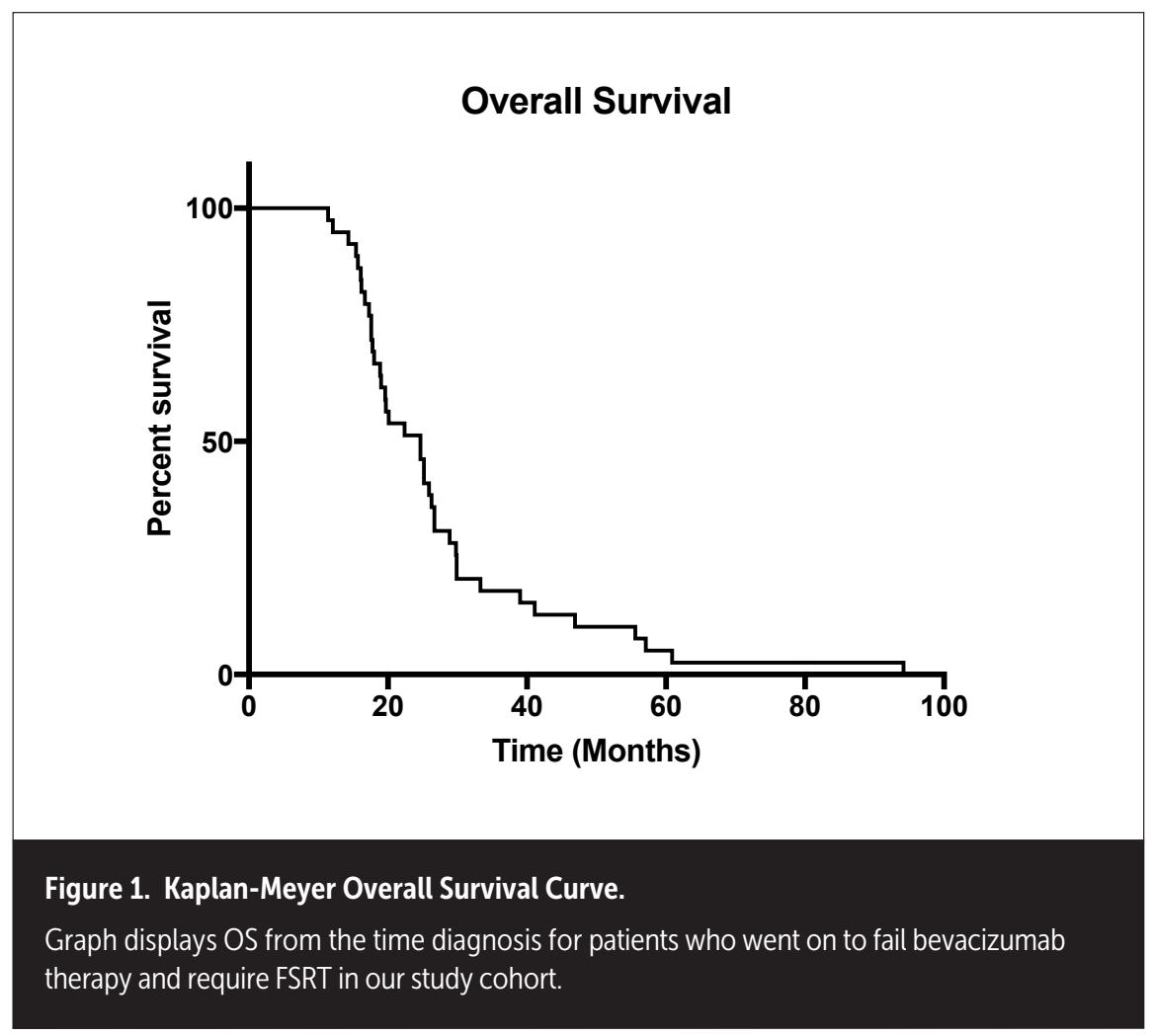

salvage FSRT was $8.5 \mathrm{~m}$ (range $2.4-32 \mathrm{~m}$ ), and the median overall survival after FSRT was $4.8 \mathrm{~m}$. Data are presented in table form (Table 2) as well as in the form of a KaplanMeyer survival curve (Figure 1).

\section{Multivariate Analysis}

Multivariate analysis was performed to investigate whether different variables in our study population influenced OS from recurrence or from FSRT therapy (Table 3). These included age at recurrence, KPS score, volume of recurrence, histology ( $A A$ vs GBM) or re-resection status. Importantly, out of all of these variables, only re-resection demonstrated a statistically significant association with overall survival from recurrence (HR 2.59; $p=0.04$ ). Additionally, there was a trend towards significance for re-resection status associated with OS from FSRT (HR $1.87 \mathrm{p}=0.17$ )

\section{Toxicity}

No patients demonstrated clinically significant acute morbidity, with no grade III or higher toxicity observed. All patients were able to complete the prescribed radiation course without interruption. There were no observed hospitalizations or surgeries for early acute or delayed toxicity in the study population.

\section{DISCUSSION}

Despite recent advances such as bevacizumab that have extended overall survival in patients with high-grade gliomas, treatment failure and disease progression while on bevacizumab remain as an unfortunate reality in managing these patients. Therefore, overall prognosis remains quite poor. Despite this clinical need, there remains a paucity of literature regarding the management of patients who fail bevacizumab. In that context, the present study investigated FSRT as a potential treatment modality to address this problem.

Prior to FDA-approval of bevacizumab, FSRT had been previously studied in the setting of recurrent HGG, with generally favorable results. Multiple studies have shown FSRT to be efficacious, with OS in these studies ranging from 5-11 months. Moreover, these studies showed FSRT to be very well tolerated, with a low rate of grade 3 toxicities, radiation necrosis (RN) and reoperation. ${ }^{10-12}$

Of note, one study (Lederman et al.) observed significantly more toxicity and reoperation (11 of 88 patients) than the others. This toxicity outlier can perhaps be explained by the use of a different dosing regimen (24 Gy in 4 fractions in Lederman et al. versus 30-35 Gy in 6-10 fractions in the other studies). ${ }^{13}$ In a head to head trial, Patel et al. compared stereotactic radiosurgery (SRS) with FSRT and showed comparable overall survival and radiographic tumor response between the two modalities, with a trend towards fewer events of radiation necrosis (RN) in the FSRT cohort. ${ }^{14}$

The data on FSRT treatment following bevacizumab failure is much more limited. In a retrospective study, Torcuator et al. looked at two cohorts of patients who failed bevacizumab: one that received either FSRT or SRS and one that received no FSRT/SRS. They demonstrated an increased overall survival in patients receiving FSRT/SRS (7.2 vs 3.3 months in untreated patients). Another study that is published only in abstract form by Nehaw et al. similarly looked at RT (including 6 patients who received FSRT) versus non-RT regimens following bevacizumab failure and showed statistically significant increased survival in the radiation group ( 8.8 vs. 5.4 months for untreated). Despite this small body of literature, neither of these studies investigated FSRT alone after bevacizumab 
Table 3. Multivariate Analysis. Multivariate analysis is shown for the study cohort. Variables were tested for association with OS from Recurrence as well as OS From FSRT. Data are expressed as Hazard Ratios (HR) and $p$-value. P-value of $<0.05$ was considered significant.

\begin{tabular}{|l|l|}
\hline OS from Recurrence Multivariate & $H R=0.99 ; p=0.67$ \\
\hline Age at Recurrence & $H R=1.27 ; p=0.61$ \\
\hline KPS $<=80$ & $H R=1.09 ; p=0.32$ \\
\hline Volume of recurrence $>50 c c$ & $H R=2.59 ; p=0.04$ \\
\hline Re-resection yes vs no & $H R=0.99 ; p=0.99$ \\
\hline Histology AA vs GBM & \\
\hline OS from FSRT Multivariate & $H R=1.01 ; p=0.70$ \\
\hline Age at Recurrence & $H R=0.73 ; p=0.53$ \\
\hline KPS $<=80$ & $>50 c c \quad H R=1.02 ; p=0.83$ \\
\hline Volume of recurrence & $H R=1.87 ; p=0.17$ \\
\hline Re-resection yes vs no & $H R=1.72 ; p=0.46$ \\
\hline Histology AA vs GBM & \\
\hline
\end{tabular}

failure, nor did they report on safety or toxicity of these approaches.

In that context, the present study represents one of the first studies to specifically investigate the role of FSRT in the context of bevacizumab failure. Indeed, our work builds off of previous work by both our group and others showing comparable benefit and improved safety in FSRT regimens for HGGs as compared to SRS for treatment of HGG in other contexts. ${ }^{10,11,13}$ Specifically, the present study demonstrates the feasibility, efficacy and tolerability of such an approach in patients who fail bevacizumab.

One limitation of our study is the lack of a control cohort for comparison in terms of outcomes to put our overall survival into context. Historically, patients who fail bevacizumab have been shown in a recent review of sixteen studies to have an overall survival of 3.8 months. ${ }^{4-6}$

Thus, our observed overall survival compares favorably to, and indeed exceeds that mark. Taken in the context of the aforementioned studies which show benefit of RT vs. no RT in bevacizumab failure, and also that FSRT and SRS provide similar OS in recurrent gliomas before bevacizumab treatment, our data are consistent with these previous studies and moreover suggest a role for FSRT in the management of patients who fail bevacizumab. Despite our findings, it is worth noting that one limitation of our study is the potential bias of our dataset in that it only includes patients who are amenable to therapy with FSRT. Therefore it is difficult to directly compare our survival data to the existing literature, given that the literature includes all patients, whether or not they are eligible for FSRT. Further head-to-head studies will be needed to evaluate FSRT versus other modalities to definitively establish a role and identify populations that would most benefit.

Notably, our multivariate analysis yielded only one variable that was associated with overall survival: re-resection status. Indeed, there is controversy in the literature regarding the prognostic value of re-resection in patients with recurrent $\mathrm{HGG}^{2}$ but our data suggest that re-resection is actually deleterious in terms of survival outcomes. However, given the retrospective nature of the current study, it is difficult to draw strong conclusions from these data, as re-resection status itself may be confounding by representing underlying patient characteristics that lead to poorer prognosis. Future studies will be needed to identify patient populations who will most benefit from an FSRT regimen.

Other limits to our study include a small patient cohort (36) as well as those shortcomings inherent to all retrospective studies including selection bias and potential treatment differences in a non-randomized study. Despite these potential drawbacks, this study represents, to our knowledge, the largest literature cohort of FSRT patients in the context of bevacizumab failure. Moreover, the dire prognosis of these patients and the paucity of data regarding their management underscores the relevance of the present study, and suggests the need for future prospective randomized trials to improve survival and positively impact the lives of patients with HGG.

\section{CONCLUSIONS}

Favorable outcomes were observed using FSRT to treat patients with recurrent HGG and the treatment was well tolerated Prospective study is warranted to further evaluate the efficacy of salvage FSRT for patients with recurrent HGG after bevacizumab failure.

\section{REFERENCES}

1. Stupp R, Brada M, van den Bent MJ, Tonn JC, Pentheroudakis G, Group EGW. High-grade glioma: ESMO Clinical Practice Guidelines for diagnosis, treatment and follow-up. Annals of oncology: official journal of the European Society for Medical Oncology 2014; 25 Suppl 3:iii93-101.

2. Palmer JD, Siglin J, Yamoah K, Dan T, Champ $\mathrm{CE}$, Bar-Ad V, et al. Re-resection for recurrent high-grade glioma in the setting of re-irradiation: more is not always better. $J$ Neurooncol 2015; 124:215-21

3. Iwamoto FM, Abrey LE, Beal K, Gutin PH, Rosenblum MK, Reuter VE, et al. Patterns of relapse and prognosis after bevacizumab failure in recurrent glioblastoma. Neurology 2009; 73:1200-6.

4. Kreisl TN, Kim L, Moore K, Duic P, Royce C, Stroud I, et al. Phase II trial of single-agent bevacizumab followed by bevacizumab plus irinotecan at tumor progression in recurrent glioblastoma. J Clin Oncol 2009; 27:740-5.

5. Norden AD, Young GS, Setayesh K, Muzikansky A, Klufas R, Ross GL, et al. Bevacizumab for recurrent malignant gliomas: efficacy, toxicity, and patterns of recurrence. Neurology 2008; 70:779-87.

6. Magnuson W, lan Robins $H$, Mohindra P, Howard S. Large volume reirradiation as salvage therapy for glioblastoma after progression on bevacizumab. Journal of neuro-oncology 2014; 117:133-9. 
7. Combs SE, Gutwein S, Thilmann C, Debus J, Schulz-Ertner D. Reirradiation of recurrent WHO grade III astrocytomas using fractionated stereotactic radiotherapy (FSRT). Strahlentherapie und Onkologi: Organ der Deutschen Rontgengesellschaft [et al] 2005; 181:768-73.

8. Combs SE, Gutwein S, Thilmann C, Huber P, Debus J, Schulz-Ertner D. Stereotactically guided fractionated re-irradiation in recurrent glioblastoma multiforme. Journal of neurooncology 2005; 74:167-71.

9. Shen X, Andrews DW, Sergott RC, Evans JJ, Curran WJ, Machtay M, et al. Fractionated stereotactic radiation therapy improves cranial neuropathies in patients with skull base meningiomas: a retrospective cohort study. Radiation oncology 2012; 7:225.

10. Fogh SE, Andrews DW, Glass J, Curran W, Glass C, Champ C, et al. Hypofractionated stereotactic radiation therapy: an effective therapy for recurrent high-grade gliomas. J Clin Oncol 2010; 28:3048-53.
11. Grosu AL, Weber WA, Franz M, Stark S, Piert $M$, Thamm R, et al. Reirradiation of recurrent high-grade gliomas using amino acid PET (SPECT)/CT/MRI image fusion to determine gross tumor volume for stereotactic fractionated radiotherapy. Int J Radiat Oncol Biol Phys 2005; 63:511-9.

12. Fokas E, Wacker U, Gross MW, Henze $M$, Encheva E, Engenhart-Cabillic R Hypofractionated stereotactic reirradiation of recurrent glioblastomas : a beneficial treatment option after high-dose radiotherapy? Strahlenther Onkol 2009; 185:235-40.

13. Lederman G, Wronski M, Arbit E, Odaimi M, Wertheim S, Lombardi E, et al. Treatment of recurrent glioblastoma multiforme using fractionated stereotactic radiosurgery and concurrent paclitaxel. Am J Clin Oncol 2000 23:155-9.

14. Patel M, Siddiqui $F$, Jin JY, Mikkelsen T, Rosenblum M, Movsas B, et al. Salvage reirradiation for recurrent glioblastoma with radiosurgery: radiographic response and improved survival. J Neurooncol 2009; 92:185-91.

\section{Corresponding Author Wenyin Shi, MD, PhD} Department of Radiation Oncology Sidney Kimmel Medical College Thomas Jefferson University Sidney Kimmel Cancer Center at Jefferson

111 South 11th Street Philadelphia, PA 19107

P: $215-955-6700$

F: 215-503-0013

E: Wenyin.Shi@jefferson.edu 